\title{
Neuronal Integration of Synaptic Input in the Fluctuation- Driven Regime
}

\author{
Alexandre Kuhn, ${ }^{1}$ Ad Aertsen, ${ }^{1}$ and Stefan Rotter ${ }^{1,2}$ \\ ${ }^{1}$ Neurobiology and Biophysics, Institute of Biology III, Albert-Ludwigs-University, D-79104 Freiburg, Germany, and ${ }^{2}$ Theory and Data Analysis, Institute \\ for Frontier Areas of Psychology and Mental Health, D-79098 Freiburg, Germany
}

\begin{abstract}
During sensory stimulation, visual cortical neurons undergo massive synaptic bombardment. This increases their input conductance, and action potentials mainly result from membrane potential fluctuations. To understand the response properties of neurons operating in this regime, we studied a model neuron with synaptic inputs represented by transient membrane conductance changes. We show that with a simultaneous increase of excitation and inhibition, the firing rate first increases, reaches a maximum, and then decreases at higher input rates. Comodulation of excitation and inhibition, therefore, does not provide a straightforward way of controlling the neuronal firing rate, in contrast to coding mechanisms postulated previously. The synaptically induced conductance increase plays a key role in this effect: it decreases firing rate by shunting membrane potential fluctuations, and increases it by reducing the membrane time constant, allowing for faster membrane potential transients. These findings do not depend on details of the model and, hence, are relevant to cells of other cortical areas as well.
\end{abstract}

Key words: synaptic integration; membrane conductance; primary visual cortex; integrate-and-fire; intracellular recording; neural coding

A growing number of laboratories record the membrane potential of cortical neurons in vivo [e.g., in the visual cortex (Pei et al., 1991; Ahmed et al., 1997; Azouz et al., 1997; Hirsch et al., 1998; Bringuier et al., 1999; Carandini and Ferster, 2000)], providing detailed insight into the way neurons operate within the functioning cortical network. These experiments showed that the barrage of synaptic input impinging on cortical neurons during sensory stimulation substantially increases the somatic input conductance (Borg-Graham et al., 1998; Hirsch et al., 1998). This, in turn, is expected to change the integration properties of the neurons (Bernander et al., 1991; Rapp et al., 1992; Destexhe and Paré, 1999; Rudolph and Destexhe, 2003). Moreover, these experiments revealed that the membrane potential strongly fluctuates [e.g., in response to visual stimuli (Anderson et al., 2000b) ] but, on average, remains below firing threshold because it combines excitation and inhibition (Borg-Graham et al., 1998). Here we show that, under these conditions, both membrane potential fluctuations and firing rate evoked by simultaneously increasing excitation and inhibition are predicted to behave nonmonotonically. In particular, they can reach their maximal amplitude at moderate synaptic input rates and decrease for higher input rates.

Neuronal firing rate is commonly assumed to be the carrier of information in the brain [but see also, e.g., for the visual cortex, Bair (1999)]. It is thus of utmost importance to understand how

Received July 16, 2003; revised Dec. 12, 2003; accepted Dec. 12, 2003.

This work was supported by the Human Frontier Science Program and by the German-Israeli Foundation for Scientific Research and Development. We thank Yves Frégnac, Idan Segev, Carl van Vreeswijk, and Thomas Wachtler for valuable comments on a previous version of this manuscript.

Correspondenceshould be addressed to Dr.Stefan Rotter, Theoryand Data Analysis, Institutefor Frontier Areas of Psychology and Mental Health, Wilhelmstrasse 3a, D-79098 Freiburg, Germany. E-mail: stefan.rotter@biologie.uni-freiburg.de.

DOl:10.1523/JNEUROSCI.3349-03.2004

Copyright $\odot 2004$ Society for Neuroscience $\quad 0270-6474 / 04 / 242345-12 \$ 15.00 / 0$ patterns of synaptic inputs determine the firing rate. To explain the irregular firing observed throughout the cortex (Softky and Koch, 1993), Shadlen and Newsome $(1994,1998)$ proposed that the firing rate of cortical neurons is essentially controlled by the size of membrane potential fluctuations: If excitation and inhibition are comodulated, such that the increase of excitation is compensated by a simultaneous increase of inhibition, the membrane potential remains constant on average; however, the amplitude of the fluctuations increases with the balanced input rates, causing the probability to hit firing threshold and, thus, firing rate, to increase (Gerstein and Mandelbrot, 1964). Moreover, because action potentials result from occasional (random) excursions of membrane potential above firing threshold, this mechanism yields irregular spiking at all rates (van Vreeswijk and Sompolinsky, 1996).

The scheme just described, however, neglects the experimentally observed change of membrane properties induced by synaptic activity (Borg-Graham et al., 1998), and in particular it does not accommodate for shunting effects. As we will see, this radically changes the response of a neuron. We studied the response of a model neuron with synaptic inputs consisting of transient conductance changes, operating in the regime in which spikes are elicited by membrane potential fluctuations. First, we investigated the neuronal response to balanced input. Specifically, we analyzed how integration of individual synaptic events, membrane potential fluctuations, and firing rate change with the level of synaptic bombardment. In a second step, we relaxed the balanced input condition and studied the neuronal response (in the fluctuation-driven regime) to arbitrary levels of excitation and inhibition. We show that the synaptically induced conductance increase shapes the neuronal response in an unexpected manner and that the model accounts for recent observations made in vivo. 
Our analysis might also be useful for inferring the level of excitation and inhibition from intracellular recordings in vivo.

Preliminary results have been presented previously in abstract form (Kuhn et al., 2002).

\section{Materials and Methods}

In this section we analyze the mean and SD of the free membrane potential (i.e., the membrane potential of the neuron without spiking) for two model neurons. In the first one, synaptic inputs are modeled as transient currents, whereas in the second one they are modeled as transient membrane conductance changes. As we show in Results, the second model can account for the input-driven membrane conductance increase observed in vivo and behaves quite differently, leading to new views on neuronal functioning. Thus, a comparison with the simpler model with currentbased synapses will permit us to highlight and better understand the specific properties of conductance input.

The analytical expression that we derived for the SD of membrane potential fluctuations for the model with conductance input is new. The parameters used in Results are listed after the analysis of each model. The last part of this section describes the firing rate model, derived on the basis of our results and used in Figure $4 c$.

Neuron with synaptic inputs modeled as current sources. The different contributions to the current flowing across the membrane are related by the equation (Jack et al., 1975):

$$
C \frac{d}{d t} U(t)+\left[U(t)-U_{r}\right] G_{l}-I(t)=0,
$$

with $U(t)$, free membrane potential, $I(t)$, synaptic current input, $C$, membrane capacitance, $G_{l}$, membrane leak conductance, and $U_{r}$, resting membrane potential. It gives rise to a linear differential equation with constant coefficients (Koch, 1999):

$$
\tau_{\mathrm{m}} \frac{d}{d t} U(t)=-\left[U(t)-U_{r}\right]+\frac{I(t)}{G_{l}} .
$$

The membrane time constant is $\tau_{\mathrm{m}}=C / G_{l}$. The current $I(t)$ is induced by excitatory and inhibitory synaptic events:

$$
I(t)=I_{e}(t)+I_{i}(t)=\sum_{j} \operatorname{EPSC}\left(t-t_{j}\right)+\sum_{k} \operatorname{IPSC}\left(t-t_{k}\right),
$$

with $\operatorname{EPSC}(t)$ and $\operatorname{IPSC}(t)$ representing individual stereotyped EPSCs and IPSCs, respectively. The times of occurrence of excitatory and inhibitory synaptic events are denoted by $t_{j}$ and $t_{k}$, respectively. They were assumed to follow Poisson statistics with rates $\lambda_{e}$ and $\lambda_{i}$, respectively. Individual EPSCs and IPSCs were modeled as $\alpha$-functions (Koch, 1999; Rotter and Diesmann, 1999):

$$
\begin{aligned}
& \operatorname{EPSC}(t)=A_{e} \frac{t}{\tau_{e}} e^{1-t / \tau_{e}} H(t) \\
& \operatorname{IPSC}(t)=A_{i} \frac{t}{\tau_{i}} e^{1-t / \tau_{i}} H(t),
\end{aligned}
$$

with $A_{e}>0, A_{i}<0$, peak synaptic currents and $\tau_{e}, \tau_{i}$, synaptic time constants.

$H(x)$ is the Heaviside step function:

$$
H(x)= \begin{cases}0 & \text { for } x<0 \\ 1 & \text { for } x \geq 0\end{cases}
$$

Because the system defined by Equation 1 is linear, knowing the membrane response to a single PSC is sufficient to derive the statistical properties of the membrane potential fluctuations caused by multiple PSCs (synaptic bombardment). The response to a single PSC is given by the solution of:

$$
\tau_{\mathrm{m}} \frac{d}{d t} U(t)=-\left[U(t)-U_{r}\right]+\frac{\operatorname{PSC}(t)}{G_{l}} \text { with } U(0)=U_{r},
$$

where $\operatorname{PSC}(t)$ is either $\operatorname{EPSC}(t)$ or $\operatorname{IPSC}(t)$. Solving for $U(t)$ gives the time course of a PSP (after subtraction of the resting potential $U_{r}$ ):

$$
\operatorname{PSP}(t)=\frac{A_{s} e}{C \tau_{s}}\left[\frac{-t e^{-t / \tau_{s}}}{1 / \tau_{s}-1 / \tau_{\mathrm{m}}}+\frac{e^{-t / \tau_{\mathrm{m}}}-e^{-t / \tau_{s}}}{\left(1 / \tau_{s}-1 / \tau_{\mathrm{m}}\right)^{2}}\right] H(t),
$$

where $\left(A_{s}, \tau_{s}\right)$ is either $\left(A_{e}, \tau_{e}\right)$ or $\left(A_{i}, \tau_{i}\right)$, defining $\operatorname{EPSP}(t)$ or $\operatorname{IPSP}(t)$, respectively. Because the PSPs superimpose linearly and the statistics of occurrence of synaptic events are assumed to be Poissonian, the mean $\mu(U)$ and variance $\sigma^{2}(U)$ of the free membrane potential are given by Campbell's theorem (Papoulis, 1991):

$$
\begin{gathered}
\mu(U)=U_{r}+\lambda_{e} \int \operatorname{EPSP}(t) d t+\lambda_{i} \int \operatorname{IPSP}(t) d t \\
\sigma^{2}(U)=\lambda_{e} \int \operatorname{EPSP}(t)^{2} d t+\lambda_{i} \int \operatorname{IPSP}(t)^{2} d t .
\end{gathered}
$$

Explicit expressions are given by:

$$
\begin{gathered}
\int \operatorname{PSP}(t) d t=A_{s} \tau_{s} e \tau_{\mathrm{m}} / C \\
\int \operatorname{PSP}(t)^{2} d t=\left(2 \tau_{\mathrm{m}}+\tau_{s}\right)\left[\frac{A_{s} \tau_{s} e \tau_{\mathrm{m}}}{2 C\left(\tau_{\mathrm{m}}+\tau_{s}\right)}\right]^{2} .
\end{gathered}
$$

Note that the dependence of the inhibitory input rate $\lambda_{i}$ on the excitatory input rate $\lambda_{e}$ under the constraint of a constant mean free membrane potential $\mu(U)$ (balanced input; see Fig. 1a) can be derived from Equation 2:

$$
\lambda_{i}=-\frac{\int \operatorname{EPSP}(t) d t}{\int \operatorname{IPSP}(t) d t} \lambda_{e}-\frac{U_{r}-\mu(U)}{\int \operatorname{IPSP}(t) d t} .
$$

For this model, any admissible pair of mean and SD of the membrane potential (Eqs. 2, 3) corresponds to a uniquely determined pair of excitatory and inhibitory input rates.

The membrane parameters used were $C=250 \mathrm{pF}, G_{l}=1 / 60 \mu \mathrm{S}$, $U_{r}=-70 \mathrm{mV}$ (McCormick et al., 1985). The synaptic parameters were chosen such that amplitude and width of the EPSP and the IPSP matched the measurements of Tarczy-Hornoch et al. $(1998,1999): A_{e}=390.5$ $\mathrm{pA}, A_{i}=-74 \mathrm{pA}, \tau_{e}=0.2 \mathrm{msec}, \tau_{i}=2 \mathrm{msec}$. Note that TarczyHornoch et al. $(1998,1999)$ measured EPSPs at $-70 \mathrm{mV}$ and IPSPs at $-60 \mathrm{mV}$. The peak synaptic current values given here are set such that the amplitude of model PSPs corresponds to the amplitude of PSPs measured at $-55 \mathrm{mV}$; i.e., model PSPs were fit to the measured and (linearly) scaled PSPs. For this scaling, the excitatory and inhibitory synaptic reversal potentials were assumed to be 0 and $-75 \mathrm{mV}$, respectively (TarczyHornoch et al., 1998, 1999). For the complete spiking model, a spike was generated whenever the membrane potential hit the firing threshold $U_{\theta}=-50 \mathrm{mV}$. To mimic the refractory period, the membrane potential was immediately reset to $U_{\text {reset }}=-60 \mathrm{mV}$ and clamped at this value for $\tau_{\text {refr }}=2 \mathrm{msec}$ (Troyer and Miller, 1997). A time step of $0.01 \mathrm{msec}$ was used for the numerical simulations.

Neuron with synaptic inputs modeled as transient conductances. The total current flow across the membrane in the case of conductance-based synapses is determined by (Koch, 1999):

$C \frac{d}{d t} U(t)+\left[U(t)-U_{r}\right] G_{l}+\left[U(t)-U_{e}\right] G_{e}(t)$

$$
+\left[U(t)-U_{i}\right] G_{i}(t)=0,
$$

where $G_{e}(t)$ and $G_{i}(t)$ are the synaptic conductances, and $U_{e}$ and $U_{i}$ are 
the synaptic reversal potentials for excitation and inhibition, respectively. This equation can be rewritten as:

$$
\tau_{\mathrm{eff}}(t) \frac{d}{d t} U(t)=-U(t)+\frac{U_{r} G_{l}+U_{e} G_{e}(t)+U_{i} G_{i}(t)}{G_{\mathrm{tot}}(t)} .
$$

We call $G_{\text {tot }}(t)=G_{l}+G_{e}(t)+G_{i}(t)$ the total membrane conductance and $\tau_{\text {eff }}(t)=C / G_{\text {tot }}(t)$ the effective membrane time constant. We use $g_{e}(t)$ and $g_{i}(t)$ to denote the membrane conductance changes elicited by a single excitatory or inhibitory synaptic event. They were modeled by $\alpha$-functions:

$$
\begin{aligned}
& g_{e}(t)=B_{e} \frac{t}{\tau_{e}} e^{1-t / \tau_{e}} H(t), G_{e}(t)=\sum_{j} g_{e}\left(t-t_{j}\right), \\
& g_{i}(t)=B_{i} \frac{t}{\tau_{i}} e^{1-t / \tau_{i}} H(t), G_{i}(t)=\sum_{k} g_{i}\left(t-t_{k}\right) .
\end{aligned}
$$

$B_{e}$ and $B_{i}$ are the peak excitatory and inhibitory synaptic conductances, respectively. The time constants of the conductance changes $\tau_{e}$ and $\tau_{i}$ were assumed to be identical to the time constants of the EPSC and IPSC, respectively, in the current-based model.

For an approximate analytical treatment of the effect of synaptic bombardment on synaptic integration, we replace $G_{e}(t)$ and $G_{i}(t)$ by their mean values $\mu\left(G_{e}\right)$ and $\mu\left(G_{i}\right)$, respectively. These are again given by Campbell's theorem (Papoulis, 1991):

$$
\begin{aligned}
& \mu\left(G_{e}\right)=\lambda_{e} \int g_{e}(t) d t=\lambda_{e} B_{e} \tau_{e} e \\
& \mu\left(G_{i}\right)=\lambda_{i} \int g_{i}(t) d t=\lambda_{i} B_{i} \tau_{i} e .
\end{aligned}
$$

An approximation of the mean membrane potential level $\mu(U)$ induced by the excitatory and inhibitory synaptic bombardment is then given by:

$$
\begin{aligned}
& \mu(U) \approx \frac{U_{r} G_{l}+U_{e} \mu\left(G_{e}\right)+U_{i} \mu\left(G_{i}\right)}{\mu\left(G_{\text {tot }}\right)}, \\
& \quad \text { with } \mu\left(G_{\text {tot }}\right)=G_{l}+\mu\left(G_{e}\right)+\mu\left(G_{i}\right) .
\end{aligned}
$$

Conversely, Equation 8 yields an approximation of the inhibitory input rate $\lambda_{i}$ needed to achieve a given constant free membrane potential level (balanced input), for any given excitatory input rate $\lambda_{e}$ :

$$
\lambda_{i} \approx-\frac{\left[U_{e}-\mu(U)\right] \int g_{e}(t) d t}{\left[U_{i}-\mu(U)\right] \int g_{i}(t) d t} \lambda_{e}-\frac{\left[U_{r}-\mu(U)\right] G_{l}}{\left[U_{i}-\mu(U)\right] \int g_{i}(t) d t} .
$$

Note that the expression above is a linear function of $\lambda_{e}$, non-negative and strictly increasing. In contrast to the current-based model, its slope is not only determined by the characteristics of excitatory and inhibitory synaptic events, but also by the membrane potential level considered. The higher the membrane potential level (i.e., farther from the inhibitory and closer to the excitatory synaptic reversal potential), the smaller the slope, such that an increase of $\lambda_{e}$ necessitates a smaller increase of $\lambda_{i}$ to maintain a constant membrane potential level.

The smaller the fluctuations of $G_{e}(t)$ and $G_{i}(t)$ are, the better is the approximation of replacing them by their mean values. The variances of $G_{e}(t)$ and $G_{i}(t)$ are given by (Papoulis, 1991):

$$
\begin{aligned}
& \sigma^{2}\left(G_{e}\right)=\lambda_{e} B_{e}^{2} \tau_{e} e^{2} / 4 \\
& \sigma^{2}\left(G_{i}\right)=\lambda_{i} B_{i}^{2} \tau_{i} e^{2} / 4 .
\end{aligned}
$$

Because these expressions scale linearly with $\lambda_{e}$ or $\lambda_{i}$, respectively, the ratios $\sigma\left(G_{e}\right) / \mu\left(G_{e}\right)$ and $\sigma\left(G_{i}\right) / \mu\left(G_{i}\right)$ are asymptotically proportional to $1 / \sqrt{\lambda_{e}}$ and $1 / \sqrt{\lambda_{i}}$, respectively. This shows that the approximation on $G_{e}(t)$ and $G_{i}(t)$ improves as the rates increase.

With the replacement of $G_{e}(t)$ and $G_{i}(t)$ by their mean values, the effective membrane time constant $\tau_{\text {eff }}(t)$ (Eq. 5) becomes $\tilde{\tau}_{\text {eff }}=$ $C / \mu\left(G_{\text {tot }}\right)$, i.e., it takes a constant value over time. Note that $\tilde{\tau}_{\text {eff }}$ is actually a first-order approximation of $\mu\left(\tau_{\text {eff }}\right)$, the mean effective time constant. Again, the smaller the fluctuations of $\tau_{\text {eff }}(t)$ are compared with $\mu\left(\tau_{\text {eff }}\right)$, the better is the approximation. A first-order estimate of the variance of $\tau_{\text {eff }}(t)$ is (Papoulis, 1991):

$$
\sigma^{2}\left(\tau_{\mathrm{eff}}\right) \approx \frac{C^{2}}{\mu\left(G_{\mathrm{tot}}\right)^{4}}\left[\sigma^{2}\left(G_{e}\right)+\sigma^{2}\left(G_{i}\right)\right] .
$$

Estimates of $\mu\left(\tau_{\text {eff }}\right)$ and $\sigma\left(\tau_{\text {eff }}\right)$ based on numerical simulations are represented in Figure $2 f$. They were in excellent agreement with the analytical approximations given here. Again, the approximation of the ratio $\sigma\left(\tau_{\text {eff }}\right) / \mu\left(\tau_{\text {eff }}\right)$ is asymptotically proportional to $1 / \sqrt{\lambda_{e}}$ and $1 / \sqrt{\lambda_{i}}$, and the approximation of $\tau_{\text {eff }}(t)$ by $\tilde{\tau}_{\text {eff }}$ becomes better with increasing input rates; however, and as shown in Results (see Figs. 2, 4), the expressions for the mean membrane potential (Eq. 8), for the PSP time course (Eq. 11), and for the variance of the membrane potential (Eq. 12) derived with the help of these approximations are in close agreement with numerical simulations at very low input rates already. Rearranging the terms in Equation 10, we can write:

$$
\frac{\sigma\left(\tau_{\text {eff }}\right)}{\tilde{\tau}_{\text {eff }}} \approx \frac{\sigma\left(G_{\text {tot }}\right)}{\mu\left(G_{\text {tot }}\right)},
$$

which means that the relative fluctuation of the effective membrane time constant around its mean is approximately equal to the relative fluctuation of the total membrane conductance around its mean.

The approximate membrane potential response to a single synaptic input in the presence of synaptic bombardment is now given by:

$$
\tilde{\tau}_{\text {eff }} \frac{d}{d t} U(t)=-[U(t)-\mu(U)]-\frac{\left[U(t)-U_{s}\right] g_{s}(t)}{\mu\left(G_{\text {tot }}\right)} \text { with } U(0)=\mu(U),
$$

where $g_{s}(t)$ and $U_{s}$ can be either $g_{e}(t)$ and $U_{e}$, or $g_{i}(t)$ and $U_{i}$, respectively. Assuming that the amplitude of a single PSP is small compared with the distance to the synaptic reversal potential, we consider the synaptic driving force $U(t)-U_{s}$ as a constant and approximate it by $\mu(U)-U_{s}$. The solution for $U(t)$ in the previous equation (now analogous to Eq. 1) gives the approximated time course of a PSP [after subtraction of $\mu(U)]$ :

$$
\operatorname{PSP}(t) \approx\left[U_{s}-\mu(U)\right] \frac{B_{s} e}{C \tau_{s}}\left[\frac{-t e^{-t / \tau_{s}}}{1 / \tau_{s}-1 / \tilde{\tau}_{\mathrm{eff}}}+\frac{e^{-t / \tilde{\tau}_{\mathrm{eff}}}-e^{-t / \tau_{s}}}{\left(1 / \tau_{s}-1 / \tilde{\tau}_{\mathrm{eff}}\right)^{2}}\right] H(t),
$$

where $\left(B_{s}, \tau_{s}, U_{s}\right)$ can be either $\left(B_{e}, \tau_{e}, U_{e}\right)$ or $\left(B_{i}, \tau_{i}, U_{i}\right)$ for $\operatorname{EPSP}(t)$ or $\operatorname{IPSP}(t)$, respectively. In addition, we assume that multiple PSPs are linearly superimposed. Again, this is a valid approximation if the membrane potential fluctuations are small compared with the distance to the synaptic reversal potential, and the driving force $U(t)-U_{s}$ does not change much in time. With this additional assumption, the variance of the free membrane potential is approximated by (Campbell's theorem):

$$
\sigma^{2}(U) \approx \lambda_{e} \int \operatorname{EPSP}(t)^{2} d t+\lambda_{i} \int \operatorname{IPSP}(t)^{2} d t
$$

It only remains to calculate the integral in the preceding expression:

$$
\int \operatorname{PSP}(t)^{2} d t \approx\left(2 \tilde{\tau}_{\text {eff }}+\tau_{s}\right)\left[\frac{\left[U_{s}-\mu(U)\right] B_{s} \tau_{s} e \tilde{\tau}_{\text {eff }}}{2 C\left(\tilde{\tau}_{\text {eff }}+\tau_{s}\right)}\right]^{2} .
$$

For very high input rates, $\tilde{\tau}_{\text {eff }}$ tends to 0 and the expression above decreases as $1 / \lambda_{e}^{2}$ and $1 / \lambda_{i}^{2}$. As a consequence, the variance of the freemembrane potential $\sigma^{2}(U)$ decreases as $1 / \lambda_{e}$ and $1 / \lambda_{i}$ (for very high 
input rates). Last, note that because $\int \operatorname{PSP}(t) d t \approx\left[U_{s}-\mu(U)\right]$ $B_{s} \tau_{s} e \tilde{\tau}_{\text {eff }} / C$, Equation 13 can be written as:

$$
\int \operatorname{PSP}(t)^{2} d t \approx\left[\int \operatorname{PSP}(t) d t\right]^{2} \frac{\left(2 \tilde{\tau}_{\text {eff }}+\tau_{s}\right)}{4\left(\tilde{\tau}_{\text {eff }}+\tau_{s}\right)^{2}} .
$$

For fixed $\mu(U), \mu\left(G_{\text {tot }}\right), \lambda_{e}$ and $\lambda_{i}, \int \operatorname{PSP}(t) d t$ is constant, and the expression above is asymptotically inversely related to $\tau_{s}$. That is, the larger the synaptic time constant, the smaller $\int \operatorname{PSP}(t)^{2} d t$ and, thus, the smaller the membrane potential fluctuations.

The values used for $C, G_{l}$, and $U_{r}$ were the same as in the currentbased model. The synaptic parameters were chosen so that the EPSP and IPSP (represented in Fig. 2, a and b, respectively) fitted the measurements of Tarczy-Hornoch et al. $(1998,1999): U_{e}=0 \mathrm{mV}, U_{i}=-75 \mathrm{mV}, B_{e}=$ $7.1 \mathrm{nS}, B_{i}=3.7 \mathrm{nS}, \tau_{e}=0.2 \mathrm{msec}, \tau_{i}=2 \mathrm{msec}$. The remaining parameters for the complete spiking neuron model were the same as for the model with synaptic current sources. Again, all numerical simulations were conducted with a time step of $0.01 \mathrm{msec}$.

Firing rate model. To illustrate the combined effect of membrane potential fluctuations and dynamics on the spiking response of the neuron (Results and see Fig. 4c), we use a simplified (firing rate) model. We assume that the probability of emitting a spike is proportional to the probability of the free membrane potential to be above firing threshold. Furthermore, the membrane potential does not change instantaneously but with a typical delay given by the effective membrane time constant. Thus, the latter also determines the time period during which (maximally) one spike can be fired. The firing rate $r$ is thus approximated by:

$$
r \approx \frac{1}{\tilde{\tau}_{\mathrm{eff}}} \int_{U_{\theta}}^{\infty} P(U) d U
$$

where $P(U)$ is the probability density function of the free membrane potential amplitude distribution (Abeles, 1991; Amit and Brunel, 1997). Note that this argument assumes that the effective membrane time constant is larger than the synaptic time constants such that it dominates the membrane potential dynamics. Assuming that the amplitude distribution of the free membrane potential is Gaussian [see, however, Kuhn et al. (2003)], the firing rate is given by:

$$
r \approx \frac{1}{2 \tilde{\tau}_{\mathrm{eff}}} \operatorname{erfc}\left[\frac{U_{\theta}-\mu(U)}{\sqrt{2} \sigma(U)}\right],
$$

where $\operatorname{erfc}(z)=1-(2 / \sqrt{\pi}) \int_{0}^{z} e^{-t^{2}} d t$ is the complementary error function.

\section{Results}

We start by briefly recalling how comodulation of excitation and inhibition was proposed to control firing rate. For that purpose, we consider the simple model neuron with synaptic inputs modeled as transient currents, similar to what was originally used by Shadlen and Newsome (1998).

\section{Comodulation of excitation and inhibition in a model neuron with current input}

The model neuron consists of a single compartment, bombarded with synaptic inputs inducing stereotyped EPSCs and IPSCs, respectively. Figure $1 a$ shows the fluctuating membrane potential resulting from the mixed synaptic bombardment (see Materials and Methods for details). Larger fluctuations are obtained by jointly increasing synaptic input rates. To better understand the behavior of the model, we consider the free membrane potential, i.e., the membrane potential of the neuron without spiking (Fig. $1 b$ ), which can be analyzed mathematically (see Materials and Methods). If the total excitatory and total inhibitory input rates (i.e., summated over all excitatory and inhibitory neurons in the input ensemble, respectively) are comodulated in a balanced a

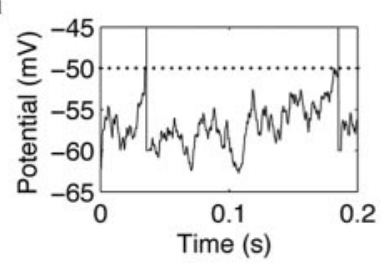

b

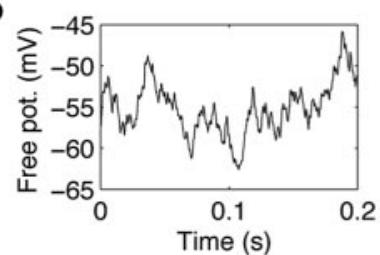

c

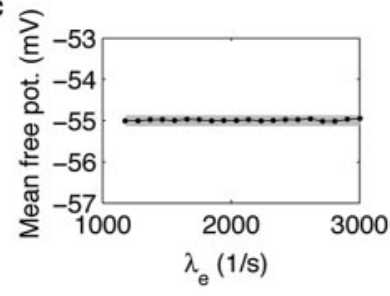

d

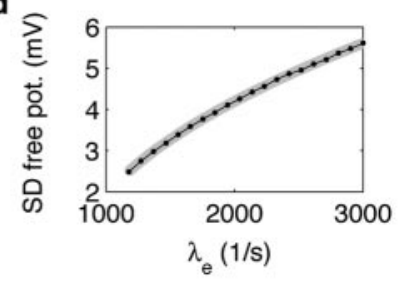

e

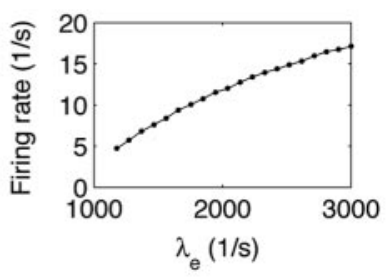

f

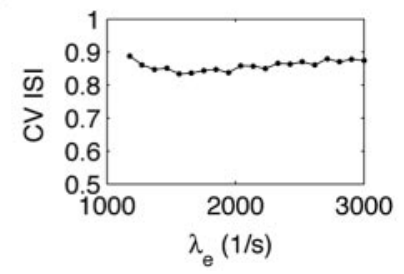

Figure 1. Free membrane potential fluctuations and firing rate of the model neuron with current input increase monotonically with the balanced increase of excitation and inhibition. $a$, Membrane potential fluctuations elicited by excitatory and inhibitory synaptic inputs with a total rate of 2000 spikes per second and 434 spikes per second, respectively. Dotted line indicates the threshold for spike generation. $b$, Free membrane potential for the same input realization as in $a . c$, Mean free membrane potential (gray line represents the analytical expression; dots depict results of numerical simulations) as a function of the excitatory input rate $\lambda_{\mathrm{e}}$. The inhibitory input rate (not shown) was covaried in a balanced manner. $d$, SD of the free membrane potential for the same input rates as in c. Again, the gray line represents the analytical expression, and dots show the results of numerical simulations. Firing rate of the model neuron $(e)$ and $C V$ of the interspike interval distribution ( $f$ ), for the same input rates as in $c$. For $c-f$, we simulated $60 \times 20$ sec of neural activity for each input condition. The statistics of interest were computed for each 20 sec trial and then averaged over all 60 trials. The SEM corresponds to the diameter of the dots or was even smaller. pot., Potential; ISI, interspike interval.

manner (Eq. 4), that is, such that the average free membrane potential remains constant (Fig. 1c, gray line, analytical expression, see Eq. 2 in Materials and Methods; black dots indicate numerical simulations), the SD of the free membrane potential increases monotonically with the excitatory synaptic input rate $\lambda_{e}$ (Fig. 1d) (Eq. 3). Likewise, the firing rate response of the spiking model (i.e., including the firing threshold and reset mechanism) to the same synaptic input increases with increasing balanced input rates (Fig. 1e), in parallel to the free membrane potential fluctuations. In addition, spike timing is irregular at all input rates, as indicated by the high coefficient of variation (CV; SD divided by the mean) of the interspike interval distribution (Fig. $1 f$ ).

\section{Comodulation of excitation and inhibition in a model neuron} with conductance input

We now contrast this behavior with the response of the neuron with conductance-based synapses. We first describe the PSPs elicited by individual synaptic inputs and then turn to the impact of background synaptic activity on individual PSPs, because this will play a key role in determining the size of membrane potential fluctuations in response to different levels of background synaptic input.

\section{EPSPs and IPSPs in the absence of background activity}

The model EPSPs and IPSPs, respectively, were adjusted to mimic the average EPSP and IPSP measured in layer 4 spiny cells of the 


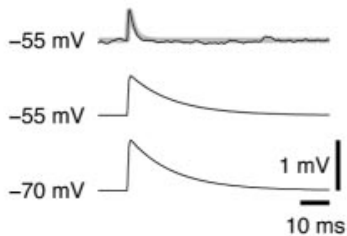

c

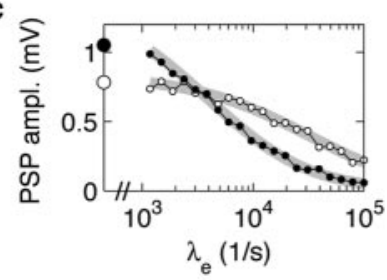

e

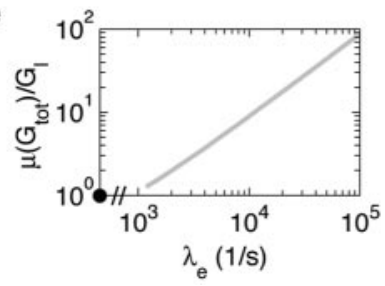

b

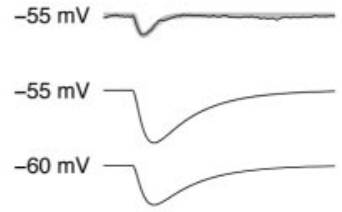

d

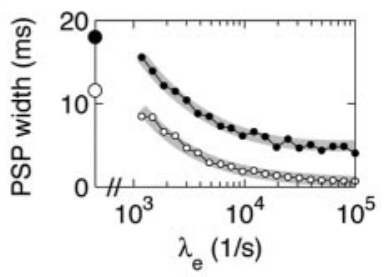

f

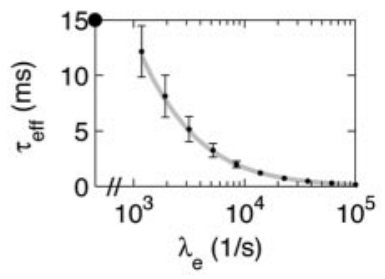

Figure 2. Amplitude and width of PSPs decrease with increasing synaptic bombardment. $a$, EPSP at a membrane potential of $-70 \mathrm{mV}$ (bottom trace) and $-55 \mathrm{mV}$ (middle trace). The top trace shows the average EPSP in the presence of synaptic background activity resulting in a mean membrane potential of $-55 \mathrm{mV}$. The thin black line represents results of numerical simulations; the gray line depicts the analytical approximation. $b$, Analogous to $a$ for the IPSP.C, PSP amplitudes as a function of the background excitatory rate $\lambda_{\mathrm{e}}$. The inhibitory input rate (not shown) was covaried with $\lambda_{\mathrm{e}}$ such that the mean free membrane potential remained constant $(-55 \mathrm{mV})$. Black lines represent numerical simulations (open circles: EPSP; solid circles: IPSP); gray lines show values for the analytically approximated PSPS. Large open and solid circles on the ordinate represent EPSP and IPSP amplitudes in the absence of synaptic background activity $\left(a, b\right.$, middle traces). $d$, Analogous to c for PSP widths. $e$, Mean membrane conductance $\mu\left(G_{\text {tot }}\right)$ relative to the leak conductance $G_{1}$. The solid circle on the ordinate indicates a relative membrane conductance of 1 , corresponding to the absence of synaptic bombardment. $f$, Effective membrane time constant $\tau_{\text {eff. }}$ Dots and error bars represent mean and SD estimated from numerical simulations. The gray line depicts the analytical approximation of the mean. The solid circle on the ordinate indicates the value of the membrane time constant with all synapses quiescent. ampl., Amplitude.

cat visual cortex in vitro (Fig. 2) (compare with Table 1 in TarczyHornoch et al., 1998 and Table 2 in Tarczy-Hornoch et al., 1999; see Materials and Methods for details of the model). The bottom trace in Figure $2 a$ represents the model EPSP at $-70 \mathrm{mV}$, the resting membrane potential (amplitude $0.998 \mathrm{mV}$; width at half amplitude $11.6 \mathrm{msec}$ ). The middle trace shows the EPSP when the membrane was depolarized to $-55 \mathrm{mV}$ by injecting a constant current. Now, the amplitude is smaller because the driving force for the corresponding ions is reduced. Analogously, the bottom trace in Figure $2 b$ shows the model IPSP at a baseline potential of $-60 \mathrm{mV}$ (amplitude $0.788 \mathrm{mV}$; width $18.0 \mathrm{msec}$ ); the middle trace depicts the IPSP when the membrane was depolarized to $-55 \mathrm{mV}$. In the latter case, the driving force for the corresponding ions was increased, and hence the IPSP had a larger amplitude.

\section{Effect of synaptic bombardment on PSPs}

To assess the effect of background activity and the associated membrane conductance increase on the integration of individual synaptic events, the model neuron was bombarded with random (Poisson) synaptic inputs. Excitatory $\left(\lambda_{e}\right)$ and inhibitory $\left(\lambda_{i}\right)$ input rates were balanced $\left(\lambda_{e}=9655\right.$ spikes per second, $\lambda_{i}=$

4473 spikes per second) such that the membrane potential fluctuated around a mean level of $-55 \mathrm{mV}$ to enable a direct comparison with the case of depolarization to $-55 \mathrm{mV}$ by constant current injection without background activity (Fig. 2ab, middle traces). The thin black lines in the top traces of Figure 2, $a$ and $b$, show the average voltage response (10,000 trials) to a single excitatory or inhibitory synaptic input, superimposed on the background activity. Observe that both amplitude and width of EPSPs and IPSPs decreased considerably compared with the case without background synaptic activity, as noted previously in other model studies (Bernander, 1993; Destexhe and Paré, 1999) and suggested from in vivo recordings (J.-F. Léger, E. A. Stern, A. Aertsen, and D. Heck, unpublished observations). The gray lines represent analytical approximations (Eq. 11), which were in excellent agreement with the numerical simulations.

To systematically study the dependence of the average EPSP and average IPSP on the intensity of the background activity, we comodulated the excitatory and inhibitory input rates such that the membrane potential fluctuated around a constant mean level of $-55 \mathrm{mV}$ (Eq. 9) (see Fig. 4a). This ensured that the synaptic driving forces were kept constant. Figure 2, $c$ and $d$, shows how amplitude and width of the average responses (10,000 trials) to a single additional excitatory (open circles) or inhibitory (solid circles) synaptic input decreased with increasing input rates. Note that only the rate $\lambda_{e}$ of excitatory inputs is shown on the abscissae. The lower bound of $\lambda_{e}$ is determined by the rate needed to maintain a mean free membrane potential of $-55 \mathrm{mV}$ in the absence of inhibitory inputs. The underlying gray lines represent the amplitude and width of the analytically approximated PSPs (Eq. 11). The agreement with the simulation results was excellent for both amplitude and width, at all levels of background activity, for both EPSP and IPSP. That is, despite the high number of trials, apparent deviations were caused by the finite size of the simulated sample and not by the nature of the analytical approximation. The reliability of the analytical approximation of the PSP is important because it is used to derive an expression for the amplitude of membrane potential fluctuations as a function of synaptic bombardment (Eq. 12) (see Fig. 4b).

\section{Fast PSPs are less vulnerable to synaptic bombardment}

Interestingly, the IPSP amplitude decreased more rapidly than the EPSP amplitude when background activity was increased (Fig. 2c). This is attributable to the different time constants of the excitatory and inhibitory synaptic conductance transients. In the extreme case of a pulse-like synaptic conductance change, the PSP amplitude (but not its decay) is only determined by the capacitive properties of the membrane. It is independent of the total membrane conductance and, hence, of the level of background activity. By contrast, if the synaptic input takes the form of a long step, the saturated amplitude of the voltage response is inversely related to the membrane conductance. Because the average membrane conductance increases linearly with the synaptic input rates (Fig. 2e) (Eqs. 6, 7), the voltage response in this case would be inversely related to the input rates. Thus, depending on the duration of the synaptic input, the PSP amplitude will be more or less affected by the membrane conductance (Koch et al., 1996; Koch, 1999) and hence by the level of background activity. In our model, the excitatory synaptic conductance transient was faster (time constant $\tau_{e}=0.2 \mathrm{msec}$ ) than that of inhibition $\left(\tau_{i}=\right.$ $2 \mathrm{msec}$ ), explaining the stronger effect of background activity on the IPSP amplitude. This effect is further illustrated for three hypothetical EPSPs having identical amplitude in the absence of background activity $\left(\tau_{e}=0.2,2,20 \mathrm{msec}\right.$ ) (Fig. $3 a$ ). Inspection 
a

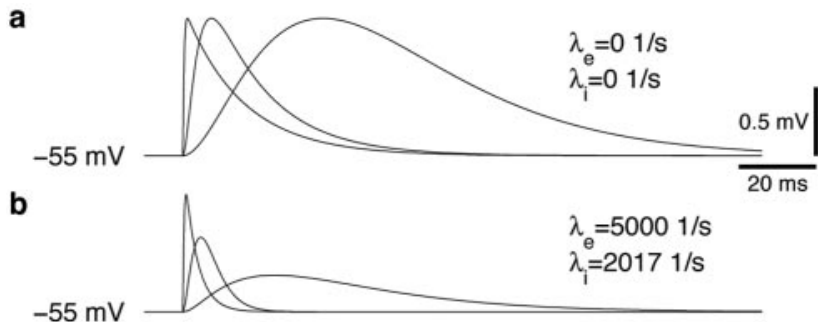

c

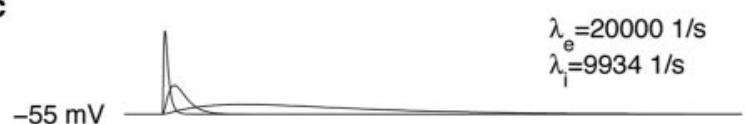

d

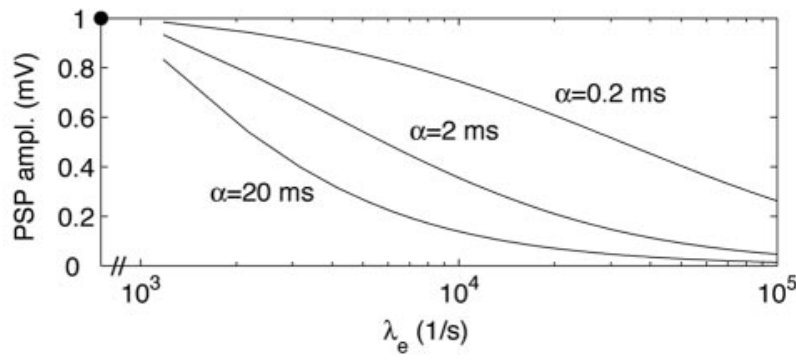

Figure 3. Amplitude of fast PSPs is less vulnerable to synaptic bombardment. $a$, Three hypothetical EPSPs with identical amplitude and different widths in the absence of synaptic background activity, at a membrane potential of $-55 \mathrm{mV}$. The synaptic conductance transients had time constants $\tau_{\mathrm{e}}=0.2,2$, and $20 \mathrm{msec}$, respectively. $b, c$, The three EPSPs for two different levels of background excitatory $\left(\lambda_{\mathrm{e}}\right)$ and inhibitory $\left(\lambda_{\mathrm{i}}\right)$ rates, each resulting in an average membrane potential of $-55 \mathrm{mV} . d$, Amplitude of the three EPSPs as a function of the background excitatory rate $\left(\lambda_{\mathrm{e}}\right)$. The background inhibitory rate (not shown) was covaried with $\lambda_{\mathrm{e}}$ such that the average membrane potential remained at $-55 \mathrm{mV}$. The solid circle on the ordinate represents the amplitude of the EPSPs in the absence of synaptic bombardment (a).

of the EPSPs at different levels of background activity (Fig. 3b,c) (excitatory and inhibitory conductances of background activity were the same as used in Fig. 2) reveals that the amplitude of the slowest EPSP experienced much more reduction than that of the fastest one (Fig. 3d). As shown in Materials and Methods and explained later in Results, the synaptic time constant also determines the detailed shape of the dependence of membrane potential fluctuations on background activity.

We now return to the decrease of PSP width with increasing background activity (Fig. 2d). It reflects the decrease of the effective membrane time constant, the latter being inversely related to the total membrane conductance (Eq. 5). A first-order analytical approximation of the mean effective membrane time constant (see Materials and Methods) is depicted by the gray line in Figure $2 f$, in very good agreement with estimates from numerical simulations (dots and error bars represent mean and SD). The error is $<0.5 \mathrm{msec}$ at the lowest input rates and improves further with increasing input rates because the relative fluctuations of the effective membrane time constant around its mean decrease with increasing input rates (see Materials and Methods). The agreement between the mean membrane time constant and its firstorder approximation is mainly responsible for the precision of the expressions for the PSP time course (Eq. 11) and for the amplitude of membrane potential fluctuations (Eq. 12; see Materials and Methods). The membrane time constant was $15 \mathrm{msec}$ in the absence of synaptic input (solid circle on the ordinate). The effective membrane time constant, however, decreased rapidly with increasing background input rates and was $<2$ msec for a a

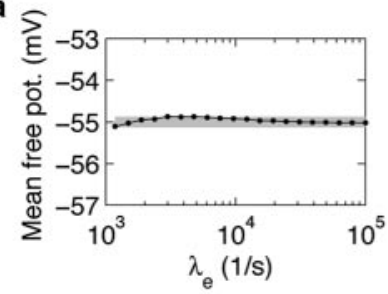

b

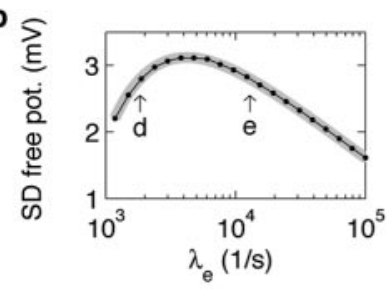

c

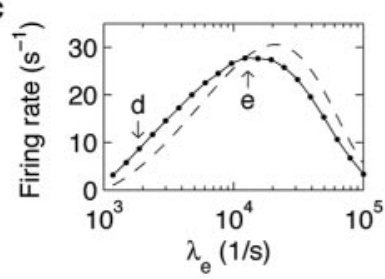

d

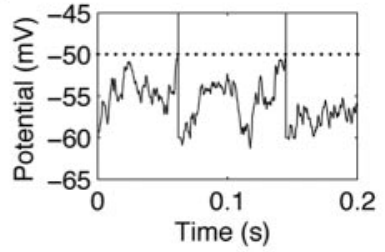

e

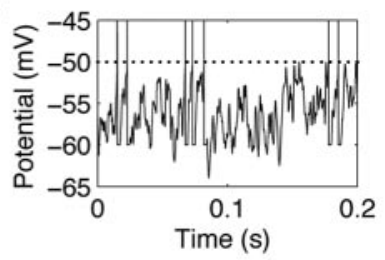

f

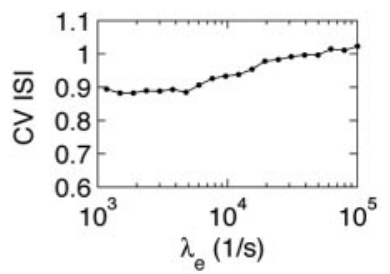

Figure 4. Free membrane potential and firing rate of the model neuron with conductance input are non-monotonic functions of the balanced increase of excitation and inhibition. Dots depict the results of numerical simulations; gray lines correspond to analytical approximations. $a$, Mean free membrane potential as a function of the excitatory rate $\lambda_{\mathrm{e}}$. The inhibitory input rate (not shown) was covaried in a balanced manner. $b$, SD of the free membrane potential for the same input rates as in $a$. Arrows indicate identical SDs, corresponding to input rates used for the simulations in $d$ and e. c, Firing rate of the neuron for the same input rates as in $a$. The dashed line corresponds to a simple firing rate model (see Results and Eq. 15). Arrows as in $c . d, e$, Time course of the membrane potential for two different levels of synaptic bombardment, resulting in equal SD of free membrane potential fluctuations, but different firing rates ( $b, c$, arrows). Dotted lines indicate the firing threshold. $f(V$ of the interspike interval distributions for the same input rates as in $a$. For $a-c$ and $f$, we simulated $50 \times 20 \mathrm{sec}$ of neural activity for each input condition. The statistics of interest were computed for each $20 \mathrm{sec}$ trial and then averaged over all 50 trials. The SEM correspond to the diameter of the dots or was even smaller.

physiologically realistic total excitatory input rate of $10^{4}$ spikes per second (see also Discussion).

\section{Membrane potential fluctuations and firing rate decrease at high levels of synaptic bombardment}

For the neuron with current-based synapses (Fig. 1), the balanced increase of excitation and inhibition always resulted in increased membrane potential fluctuations and thereby increased discharge rates; however, we just showed that for the model with conductance-based synapses, PSPs became smaller and shorter because of the increase in membrane conductance induced by background activity (Fig. 2). How does this effect influence the amplitude of membrane potential fluctuations and the firing rate at different levels of synaptic bombardment?

To study this question, we comodulated the excitatory and inhibitory synaptic input rates such that the mean free membrane potential remained constant (Fig. 4). We varied $\lambda_{e}$ from 1178 to 100,000 spikes per second and concomitantly, $\lambda_{i}$ from 0 to 52,149 spikes per second (Figs. $2 c-f, 3 d$ ). The mean free membrane potential (Fig. $4 a$, dots represent the results of numerical simulations) matched the targeted constant depolarization level ( -55 $\mathrm{mV}$; gray line) very well. Note that the small apparent deviation for $\lambda_{e}<20,000$ spikes per second (maximal deviation in the order 
of $0.1 \mathrm{mV}$ ) is caused by the assumption of constant excitatory and inhibitory synaptic conductances used to derive the input rates satisfying the balanced condition (Eq. 9). In agreement with our analysis (see Materials and Methods), however, the approximation improves with increasing input rates.

In marked contrast to the behavior of the model with current input, the fluctuations amplitude (Fig. 4b) did not increase monotonically with the balanced increase of excitation and inhibition: The SD first increased with increasing input rates, reached a maximum $\left(\sim 3.1 \mathrm{mV}\right.$ for $\lambda_{e} \approx 4200$ spikes per second and $\lambda_{i} \approx$ 1600 spikes per second) and decreased for higher input rates. The gray line in Figure $4 b$ represents an analytical approximation (Eq. 12) for the SD of the free membrane potential. Fluctuations were approximated by a linear superposition of EPSPs and IPSPs, taking into account their dependence on the level of synaptic bombardment (Fig. 2). This approximation was excellent over the entire range of input rates (errors are $<0.05 \mathrm{mV}$ ). Thus, we can summarize the dependence of membrane potential fluctuations on balanced input as follows. At low input rates, the joint increase of excitation and inhibition causes larger membrane potential fluctuations, similar to what has been described for the model with current input (Fig. 1); however, increasing synaptic activity also increases the membrane conductance, leading to smaller and shorter PSPs (Fig. 2c,d). Beyond a given level of background activity, the increased membrane conductance overcompensates the increase of fluctuations caused by the higher input rates, and the membrane potential fluctuations effectively decrease. By contrast, imposing a fixed high membrane leak conductance in the current-based model neuron (to mimic the high input conductance observed in cortical neurons in vivo) would not lead to the same non-monotonic effect. Indeed, it is the strong dependence of the membrane conductance on the input activity that creates the non-monotonic effect. Furthermore, it is important to realize that the decrease of membrane potential fluctuations at higher input rates is not a consequence of any particular choice of parameters. In fact, the fluctuations are bound to decrease because the shunting effect provided by the increased membrane conductance always dominates for high enough input rates (at high input rates, the variance of the free membrane potential is asymptotically inversely proportional to the excitatory input rate; Eq. 12). The details of the non-monotonic profile, however, depend on the synaptic parameters. The decrease of membrane potential fluctuations at higher balanced input rates, in particular, is weaker for faster synaptic conductance transients (data not shown; see Materials and Methods).

The effect just described is highly relevant to cortical neurons in vivo. First, maximal amplitude fluctuations were reached for input rates typical of cortical neurons. Assuming that the number of active synapses on a cortical neuron is between $10^{3}$ and $10^{4}$, maximal membrane potential fluctuations would be induced by individual input rates of the order of one spike per second (Fig. $4 b$ ), typical for ongoing activity of neocortical neurons. Second, the membrane conductance leading to maximal membrane fluctuations was approximately fivefold the leak conductance (Fig. $2 e)$, i.e., at the lower bound of what is expected for cortical neurons in vivo. Borg-Graham et al. (1998), Hirsch et al. (1998), and Anderson et al. (2000a, 2001) consistently reported that input conductance during visual stimulation was approximately three times as large as without visual stimulation (resting conductance). Moreover, ongoing network activity in the absence of visual stimulation (Arieli et al., 1996) gives rise to synaptic input and hence contributes to the resting conductance (Paré et al., 1998). In a model study, Destexhe and Paré (1999) estimated that the total conductance of parietal pyramidal neurons during periods of elevated activity was as much as 7-30 times larger than the leak conductance. Taken together, these figures indicate that the conductance increase in vivo is of the same magnitude or even larger than the conductance increase needed to observe the effect described here.

Similar to the membrane potential fluctuations, the neuronal rate response was also found to be a non-monotonic function of the balanced input rates. (Fig. $4 c$, dots represent the results of numerical simulations.) It first increased with increasing input rates, reached a maximum ( 28 spikes per second for $\lambda_{e} \approx 13,000$ spikes per second and $\lambda_{i} \approx 6,200$ spikes per second), and decreased for higher input rates. As a consequence, comodulation of excitation and inhibition does not implement a straightforward way of controlling the neuronal firing rate, as initially proposed by Shadlen and Newsome (1998).

\section{Impact of the effective membrane time constant on the firing rate}

Strikingly, the maximal firing rate response occurred for much larger input rates than the maximal fluctuation amplitude. For intermediate synaptic input activity (i.e., for input rates higher than the rates resulting in maximal membrane potential fluctuations, but lower than the rates for which the firing rate reached its maximum), increasing input rates decreased the membrane potential fluctuations but, paradoxically, increased the firing rate. We conclude that the size of the membrane potential fluctuations alone does not determine the discharge rate of the neuron.

To investigate this point, we compared the time course of the membrane potential for two different levels of synaptic bombardment, resulting in identical fluctuation amplitudes (Fig. $4 d, e$ ) (with $\lambda_{e}=1837$ spikes per second, $\lambda_{i}=348$ spikes per second, and $\lambda_{e}=12,857$ spikes per second, $\lambda_{i}=6163$ spikes per second, respectively). Despite the identical SD of the free membrane potential $(2.8 \mathrm{mV}$ ) (Fig. $4 b$, arrows), however, the firing rate was much higher in the case of higher input rates (28 vs 9 spikes per second) (Fig. $4 c$, arrows), because of the faster transients of the membrane potential. Considering that fluctuations result from the superposition of PSPs, these faster dynamics reflect the decrease of PSP width with increasing background activity (Fig. 2d).

Thus, the membrane time constant is another very effective modulator of the firing rate. In the two situations just described, the probabilities of the free membrane potential to be above threshold are practically identical because the (approximately Gaussian) distributions of free membrane potential have identical mean and SD. Nevertheless, the time for the membrane potential to hit the firing threshold again after the occurrence of a spike is shorter in the case of higher synaptic bombardment, because the membrane potential fluctuates more rapidly. As a consequence, the output firing rate is increased. The greatly decreased effective membrane time constant caused by intense synaptic bombardment (Fig. $2 f$ ) thus can be thought of as allowing for "faster sampling" of the distribution of the membrane potential. To test this idea quantitatively, we calculated the probability of the free membrane potential to be above firing threshold, normalized by the typical time scale of the membrane potential dynamics, the mean effective membrane time constant (Eq. 14). The resulting approximation (Eq. 15) is represented by a dashed line in Figure $4 c$. Despite the simplicity of this ad hoc model, the firing rate response of the spiking model neuron was replicated with unexpected accuracy. This emphasizes that both the ampli- 
tude characteristics and the dynamical properties of the membrane potential must be considered to explain the firing characteristics of the neuron; neither of the two alone suffices.

In summary, in the fluctuation-driven regime, the increased membrane conductance affects the firing rate simultaneously in two opposite ways. It increases the firing rate by allowing faster transients, and decreases it by shunting the membrane potential fluctuations. Finally, note that the firing of the spiking model with conductance input was slightly more irregular (Fig. $4 f$ ) than for the model with current input (Fig. $1 f$ ), reaching a value of 1 (corresponding to the irregularity of a Poisson process) for high input rates.

Let us point out that the balanced increase of input activity that we studied so far was defined as the combination of excitatory and inhibitory rate increases evoking no change of mean free membrane potential. This is in (apparent) contrast to studies in which balanced input referred to identical excitatory and inhibitory firing rates (Shadlen and Newsome, 1994, 1998). Our definition of balanced input, however, generalizes this situation and allows us to single out and compare the specific response of membrane potential fluctuations in model neurons with current input and conductance input.

\section{Independently varied excitatory and inhibitory input rates}

Relaxing the balanced input condition imposed so far, we varied the excitatory and inhibitory input rates independently and compared the distribution of the free membrane potential and the firing rate with the corresponding quantities for the model with current-based synapses (Fig. 5 ). Figure $5 a$ shows the analytical approximation for the mean free membrane potential for $\lambda_{e}$ and $\lambda_{i}$ ranging from 10 to 100,000 spikes per second. Input rates were constrained such that the mean free membrane potential remained between $-70 \mathrm{mV}$ (dark blue) and $-50 \mathrm{mV}$ (dark red). The values for the analytical approximation were checked against the results of numerical simulations (data not shown); the agreement was excellent over the whole input domain. Figure $5 b$ shows the mean free membrane potential of the model with current input. Here, input rates ranged from 10 to 10,000 spikes per second only, for reasons discussed later. Note that for this model, the range of input rates evoking values of the mean free membrane potential between $-70 \mathrm{mV}$ and $-50 \mathrm{mV}$ was much smaller than for the neuron with conductance input. For $\lambda_{e}=10,000$ spikes per second for instance, $\lambda_{i}$ could take values in the range of $4448-5277$ spikes per second for the model with current input, whereas it extended from 3175 to 26,865 spikes per second for the model with conductance input (Fig. $5 a$ ). This larger input range

\section{Current input}
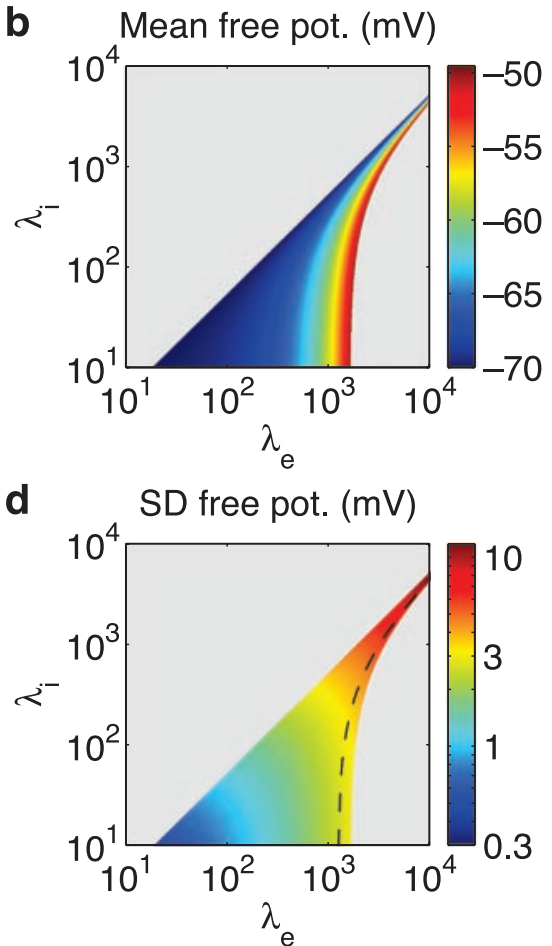

f

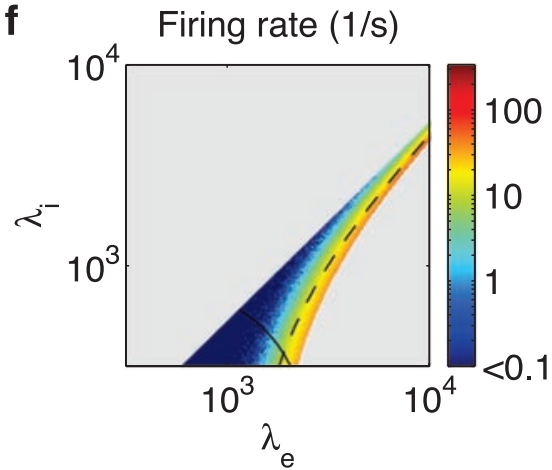

Figure 5. Free membrane potential and firing rate as a function of excitatory $\left(\lambda_{\mathrm{e}}\right)$ and inhibitory $\left(\lambda_{\mathrm{i}}\right)$ input rates, for the model neuron with conductance input (left) and with current input (right). $a$, Mean free membrane potential of the model with conducthe different axes, compared with a.c, SD of the free membrane potential for the model with conductance input. Values are shown for the same input domain as in $a$. The dashed line indicates the $-55 \mathrm{mV}$ contour of the mean free membrane potential. $d$, constrained to rates $>10^{2.5}$ spikes per second. The dashed line is again the $-55 \mathrm{mV}$ contour; the solid line indicates input rates corresponding to constant free membrane potential fluctuations ( $2.8 \mathrm{mV} \mathrm{SD}$ ). $f$, Analogous to $\mathrm{e}$ for the neuron with current input. The solid line represents input rate values corresponding to $4 \mathrm{mV} \mathrm{SD}$ of the free membrane potential.

is a direct consequence of the marked PSP dependence on synaptic background activity (Fig. 2).

The approximated SD of the free membrane potential for the model with conductance input took values from $\sim 0.3 \mathrm{mV}$ (dark blue) to $>3 \mathrm{mV}$ (yellow), with the largest fluctuations occurring for intermediate input rates (Fig. $5 c$ ). Again, the agreement with numerical simulations was excellent (data not shown). By contrast, the maximal fluctuation amplitude for the model with current input was much larger ( $>10 \mathrm{mV} \mathrm{SD}$ ) (Fig. $5 d$ ), although the maximal input rates were one order of magnitude smaller in this case. Moreover, fluctuations now were largest for high input rates, because the dependence of the membrane conductance on the synaptic bombardment and the consecutive modulation of 
PSPs were not taken into account. Note that the synaptic rates were limited to at most 10,000 spikes per second for the model with current input (Fig. $5 b, d, f$ ), precisely because the membrane potential fluctuations became unrealistically large for higher input rates. The black dashed lines in Figure 5, $c$ and $d$, are the -55 $\mathrm{mV}$ contours for the mean free membrane potential (compare with Fig. 5, $a$ and $b$, respectively); they correspond to input rates and fluctuation amplitudes as shown in Figures $4 b$ and $1 d$, respectively. Importantly, the dependence of the fluctuation amplitude along such a contour for the model with synaptic conductances (Fig. $5 c$ ) was non-monotonic at all membrane potential levels, demonstrating the generality of the non-monotonic fluctuation profile derived in Figure $4 b$. Note that the $-55 \mathrm{mV}$ contours (and only those) were equal for both models. This is because of the choice of synaptic parameters for the model with current input. Synaptic currents were chosen equal to the PSCs of the model with conductance input with the membrane potential clamped at $-55 \mathrm{mV}$ (see Materials and Methods); however, alternative parameter values for the synaptic currents would not alter the qualitative appearance of Figure $5 d$.

The firing rates of the neurons with conductance input and current input are shown in Figure 5, $e$ and $f$, respectively (input domains are reduced compared with $a$ and $b$ ). The black dashed lines again indicate the $-55 \mathrm{mV}$ contours and correspond to firing rate responses shown in Figures $4 c$ and $1 e$. For the model with conductance input (Fig. $5 e$ ), the dependence of firing rate on input rates along any mean free membrane potential contour was always non-monotonic, implying that the non-monotonic rate response (Fig. $4 c$ ) occurs for any level of the mean membrane potential.

\section{Spiking only occurs when the mean membrane potential is close to threshold}

Interestingly, the model with conductance input (Fig. 5e) only responded to inputs evoking mean free membrane potentials close to threshold. This is in marked contrast to the model with current input. There, for $\lambda_{e}=10,000$ spikes per second, for instance, the firing rate reached appreciable values for the entire range of $\lambda_{i}$, including high values giving rise to mean free membrane potentials far below threshold (Fig. $5 f$ ). The reason is that in the model with current input, the membrane potential fluctuations increase monotonically with input rates and can reach very high amplitudes. Therefore, the membrane potential can cross firing threshold even if it has, on average, a very low value, provided the input rates are high enough. Nevertheless, even for high input rates and large membrane potential fluctuations, the firing rate of the model with current input was approximately one order of magnitude smaller than for the more realistic neuron model with conductance input (Fig. $5 e, f$ ) because of its fixed membrane time constant.

The black solid line in Figure $5 e$ indicates values of the input rates evoking a constant SD of the free membrane potential $(2.8$ $\mathrm{mV}$ ) (compare Fig. $5 c$ ). The curve intersected the $-55 \mathrm{mV}$ potential contour (dashed line) twice (corresponding to input rates indicated by arrows in Figure $4 c$ ). Similarly, the black solid line in Figure $5 f$ represents input rates leading to a constant SD $(4 \mathrm{mV})$ for the model with current input. Note, however, that in this case the curve intersected the $-55 \mathrm{mV}$ potential contour only once. Indeed, for the model with current input, a pair of mean and SD of the free membrane potential corresponds to a unique pair of input rates (Eqs. 2, 3), in contrast to the model with conductance input. This has important practical consequences for the infer- ence of synaptic input conditions from the membrane potential statistics measured during in vivo recordings (see Discussion).

\section{Discussion}

Our analysis predicts that the synaptically induced conductance change occurring in cortical neurons critically shapes their response properties. Membrane potential fluctuations do not increase monotonically with increasing balanced input rates; instead, they decrease for higher input rates (Fig. $4 b$ ). Functionally even more important, the firing rate is also a non-monotonic function of balanced input (Fig. 4c), but it does not follow the same profile as the fluctuations do. The spike rate further increases with increasing balanced input, although the fluctuations had already passed their maximum and actually decrease. This difference is caused by the reduced effective membrane time constant, which allows fluctuations to cross spike threshold more often. These effects are robust and depend neither on the membrane potential level nor on the exact values of model parameters (see Results) or the shape of conductance transients. Rectangular synaptic conductance transients, for instance, led to similar results (data not shown).

Furthermore, we relaxed the balanced input condition and studied membrane potential and firing rate response over a large range of excitatory and inhibitory input rates (Fig. 5). We showed that membrane potential fluctuations already reach their maximal amplitude at moderate input rates. A further increase of excitation and inhibition results in decreasing fluctuations, even if the input is not exactly balanced.

The EPSP and IPSP shapes modeled here (Fig. 2a,b) fitted measurements on spiny cells of the visual cortex in vitro (TarczyHornoch et al., 1998, 1999). In this preparation, and for membrane potential levels close to firing threshold, the average IPSP was found to be larger and longer than the average EPSP. With increasing background activity, however, we predict that IPSPs become smaller than EPSPs (Fig. 2c), because PSPs with longer synaptic conductance transients are more vulnerable to background activity (Fig. 3). This prediction can be tested experimentally by averaging the membrane potential excursions, triggered on the spikes of excitatory or inhibitory afferent neurons (Matsumura et al., 1996) for different levels of background activity. Similarly, the AMPA component of a glutamatergic synapse is predicted to be less affected by increasing synaptic bombardment than its (slower) NMDA component.

\section{Physiological relevance}

Our model is in quantitative agreement with a number of physiological characteristics of V1 neurons. Anderson et al. (2000b) measured membrane potential fluctuations between 3 and $4 \mathrm{mV}$ (SD, averaged across cells), slightly varying with stimulus contrast and orientation. In the present model, fluctuations reached a maximal SD of $\sim 3 \mathrm{mV}$ (Figs. $4 b, 5 c$ ), which is within the range of values observed in vivo. Note that the SD of experimental data was calculated after removing action potentials. Therefore, a comparison with the free membrane potential fluctuations predicted by our model is only appropriate if the probability to be above firing threshold is low. This is generally the case for visual neurons (Anderson et al., 2000b). Moreover, in the same study, it was shown that the amplitude of fluctuations (averaged across the duration of visual trials and across cells) did not change much with stimulus contrast or orientation (Monier et al., 2003). This may be attributable to the fact that the range of synaptic input to visual cortical neurons during sensory stimulation corresponds to the range where the increased input rate is effectively compen- 
sated by the shunt because of the increased conductance, such that fluctuations remain approximately constant. Note that this range can be quite large. For a mean membrane potential of -55 $\mathrm{mV}$, for instance (Fig. $4 b$ ), the input domain for which the fluctuations in our model remained between 2.5 and $3 \mathrm{mV}$ (SD) ranged from $<2000$ to $>20,000$ spikes per second for $\lambda_{e}$.

Finally, we found that because of the limited amplitude of fluctuations, the neuron model with conductance input generated spikes at high membrane potential levels only (with $<10 \mathrm{mV}$ distance to firing threshold) (Fig. 5e), regardless of the absolute level of synaptic bombardment. This is in clear contrast to the model neuron with current input (Fig. $5 f$ ) but in good agreement with experimental studies. Carandini and Ferster (2000), and Anderson et al. (2000b) measured the firing rate as a function of the mean membrane potential (across different stimulus conditions) and showed, indeed, that visual cortical neurons only started to produce spikes at an average membrane potential level $<10 \mathrm{mV}$ below firing threshold.

\section{Functional relevance}

Several models of cortical function rely on the so-called "divisive" effect provided by the neuronal input conductance (invoked, for instance, to explain the saturation of simple cell spike response amplitude with increasing contrast) (Carandini and Heeger, 1994). More recent studies showed that input conductance actually has a subtractive effect on the input-output relationship of a neuron (Holt and Koch, 1997; Chance et al., 2002); however, the increased background synaptic input not only results in increased input conductance but also leads to larger synaptic current fluctuations. Chance et al. (2002) showed that the combination of these two effects implies that increased background synaptic input has a purely divisive effect on neuronal output. This is in apparent contrast to our results. We showed that for a fairly large range of balanced input rates, the neuronal response first increases with the input rates (Fig. $4 c$ ). This difference originates in the different input regime studied by Chance et al. (2002). Whereas the neuron in our study was driven by membrane potential fluctuations and otherwise kept in balance, Chance et al. (2002) used large depolarizing currents $(>0.5 \mathrm{nA})$ driving the membrane potential toward firing threshold. The effects of the membrane conductance increase differ in both situations. In the case of Chance et al. (2002), the shunt effect provided by the conductance increase on the driving (excitatory) current is effectively compensated by the reduced membrane time constant. In contrast, when the synaptic current is on average subthreshold (and we do not inject any additional current), the effect of the membrane conductance increase depends on its absolute level. For a small conductance increase, the decreased membrane time constant dominates and the firing rate increases (Fig. 4). For a large conductance increase, however, the shunt of membrane potential fluctuations overcompensates the effect of the decreased membrane time constant such that the firing rate eventually decreases. The interplay of these two opposite effects leaves a non-monotonic effect of input conductance on neuronal output. In conclusion, modulation of membrane conductance has quite different effects on the response properties of the neuron, depending on its precise input regime. Note that the question of the actual input regime of cortical neurons is still open. Although visual cortical neurons appear to operate in the fluctuationdriven regime during large parts of the sensory response (BorgGraham et al., 1998), excitation and inhibition can also be transiently decoupled, working in a push-pull manner (Hirsch et al., 1998).
Two recent experimental studies stressed the importance of fast membrane potential transients for the amplitude of the spike response. First, Azouz and Gray (1999) recorded the membrane potential of V1 neurons during visual stimulation and found that the number of evoked spikes was correlated with the power of the membrane potential in the $\gamma$ band $(20-70 \mathrm{~Hz})$. Second, Volgushev et al. (2002) showed that optimally oriented gratings evoked stronger fluctuations of the membrane potential in the $\gamma$ frequency range than did the nonoptimally oriented gratings. Both groups proposed that this change of spectral content was produced by a rearrangement of the fine temporal structure of the input. Our results suggest that this difference could also be explained by the altered electrical properties of the membrane. With optimally oriented gratings, as both excitation and inhibition increase (Anderson et al., 2000a), the membrane time constant decreases. Thus, faster membrane potential transients could be caused by the concomitant increase of excitation and inhibition as well as a change in precise timing.

Last, we point out that the non-monotonic response of neurons driven by balanced input may also be expected to critically determine the ensemble activity in recurrent networks. In fact, for suitable network architectures, the particular input-output relation shown here would even predict the existence of stable self-sustained network activity in the absence of external inputs.

\section{Analysis of excitation-inhibition balance in vivo}

It is a major endeavor of cortical physiology to understand how neuronal receptive fields emerge. The interplay of excitation and inhibition is an important factor in shaping the responses of cortical neurons (Moore and Nelson, 1998; Ferster and Miller, 2000). To study such questions, the inference of the input of a single neuron from its response (to a sensory stimulus) appears promising. In this context, methods based on conductance measurement were proposed to infer the relative increase of excitation and inhibition during a visual response (Anderson et al., 2000a; Borg-Graham, 2001). Here, we derived analytical approximations relating excitatory and inhibitory synaptic input rates to the mean and variance of the free membrane potential (Eqs. 8, 12) (Fig. 5). These expressions can be used reversely to directly infer the level of excitation and inhibition from membrane potential recordings, without having to actually measure the input conductance. We also found, however, that certain combinations of mean and SD of the membrane potential can be the result of two different pairs of excitatory and inhibitory input rates (Fig. $5 e$ ). This ambiguity can be resolved by exploiting second-order temporal properties of the membrane potential (e.g. its autocorrelation function), because these are differentially affected by the two candidate input conditions. Note that the inference method proposed here is limited, of course, by the assumptions made about the neuron and the input (see below). In addition, the relatively weak dependence of membrane potential fluctuations on input activity changes for certain input ranges (see above) might limit the sensitivity of the method.

\section{Limitations of the model}

Several features of cortical neurons were not taken into account in our model study. First, in reality, individual neuronal activities are not statistically independent (e.g., in the visual cortex) (Das and Gilbert, 1999), and hence a postsynaptic neuron is expected to receive partially correlated inputs. Our present knowledge of the interaction structure of synaptic input ensembles in vivo is quite sparse, however, and results derived with ad hoc correlation models have to be considered with caution (Kuhn et al., 2003). 
Second, real synapses do not respond to individual presynaptic events in a stereotypical fashion: they show dynamics and plasticity on several different time scales. Although the plasticity of excitatory and inhibitory synapses onto layer 4 spiny neurons in V1 (modeled in this study) was investigated in vitro (TarczyHornoch et al., 1998, 1999), very little is known about synaptic plasticity in vivo. Third, real neurons use voltage-gated ion channels to generate action potentials, presenting a source of nonlinear properties near spike threshold. Tiesinga et al. (2000) studied a neuron model with Hodgkin-Huxley currents and found that with current input the firing rate increased with increasing current variance, whereas the opposite was the case for the model with conductance input. This is very much in line with our findings; however, they did not ensure that the mean membrane potential was constant for different levels of synaptic input, and spikes could have been elicited by membrane potential drifts rather than by fluctuations. Finally, real cortical neurons are spatially extended, allowing for additional nonlinear processing of synaptic inputs (Holmes and Woody, 1989; Koch et al., 1990; Bush and Sejnowski, 1994; London et al., 1999; Häusser et al., 2000; Reyes, 2001). The impact of dendritic distribution of synapses and of the presence of membrane nonlinearities (including subthreshold membrane conductances) on the phenomena described here are currently being investigated.

\section{References}

Abeles M (1991) Corticonics: neural circuits of the cerebral cortex. Cambridge: Cambridge UP.

Ahmed B, Allison JD, Douglas RJ, Martin KAC (1997) An intracellular study of the contrast-dependence of neuronal activity in cat visual cortex. Cereb Cortex 7:559-570.

Amit DJ, Brunel N (1997) Model of global spontaneous activity and local structured activity during delay periods in the cerebral cortex. Cereb Cortex 7:237-252.

Anderson JS, Carandini M, Ferster D (2000a) Orientation tuning of input conductance, excitation, and inhibition in cat primary visual cortex. J Neurophysiol 84:909-926.

Anderson JS, Lampl I, Gillespie DC, Ferster D (2000b) The contribution of noise to contrast invariance of orientation tuning in cat visual cortex. Science 290:1968-1972.

Anderson JS, Lampl I, Gillespie DC, Ferster D (2001) Membrane potential and conductance changes underlying length tuning of cells in cat primary visual cortex. J Neurosci 21:2104-2112.

Arieli A, Sterkin A, Grinvald A, Aertsen A (1996) Dynamics of ongoing activity: explanation of the large variability in evoked cortical responses. Science 273:1868-1871.

Azouz R, Gray CM (1999) Cellular mechanisms contributing to response variability of cortical neurons in vivo. J Neurosci 19:2209-2223.

Azouz R, Gray CM, Nowak LG, McCormick DA (1997) Physiological properties of inhibitory interneurons in cat striate cortex. Cereb Cortex 7:534-545.

Bair W (1999) Spike timing in the mammalian visual system. Curr Opin Neurobiol 9:447-453.

Bernander Ö (1993) Synaptic integration and its control in neocortical pyramidal cells. $\mathrm{PhD}$ thesis, California Institute of Technology.

Bernander Ö, Douglas RJ, Martin KAC, Koch C (1991) Synaptic background activity influences spatiotemporal integration in single pyramidal cells. Proc Natl Acad Sci USA 88:11569-11573.

Borg-Graham LJ (2001) The computation of directional selectivity in the retina occurs presynaptic to the ganglion cell. Nat Neurosci 4:176-183.

Borg-Graham LJ, Monier C, Frégnac Y (1998) Visual input evokes transient and strong shunting inhibition in visual cortical neurons. Nature 393:369-373.

Bringuier V, Chavane F, Glaeser L, Frégnac Y (1999) Horizontal propagation of visual activity in the synaptic integration field of area 17 neurons. Science 283:695-699.

Bush PC, Sejnowski TJ (1994) Effects of inhibition and dendritic saturation in simulated neocortical pyramidal cells. J Neurophysiol 71:2183-2193.
Carandini M, Ferster D (2000) Membrane potential and firing rate in cat primary visual cortex. J Neurosci 20:470-484.

Carandini M, Heeger DJ (1994) Summation and division by neurons in primate visual cortex. Science 264:1333-1336.

Chance FS, Abbott LF, Reyes AD (2002) Gain modulation from background synaptic input. Neuron 35:773-782.

Das A, Gilbert CD (1999) Topography of contextual modulations mediated by short-range interactions in primary visual cortex. Nature 399:655-661.

Destexhe A, Paré D (1999) Impact of network activity on the integrative properties of neocortical pyramidal neurons in vivo. J Neurophysiol 81:1531-1547.

Ferster D, Miller KD (2000) Neural mechanisms of orientation selectivity in the visual cortex. Annu Rev Neurosci 23:441-471.

Gerstein GL, Mandelbrot B (1964) Random walk models for the spike activity of a single neuron. Biophys J 4:41-68.

Häusser M, Spruston N, Stuart GJ (2000) Diversity and dynamics of dendritic signaling. Science 290:739-744.

Hirsch JA, Alonso J-M, Reid RC, Martinez LM (1998) Synaptic integration in striate cortical simple cells. J Neurosci 18:9517-9528.

Holmes WR, Woody C (1989) Effects of uniform and non-uniform synaptic "activation-distributions" on the cable properties of modeled cortical pyramidal neurons. Brain Res 505:12-22.

Holt GR, Koch C (1997) Shunting inhibition does not have a divisive effect on firing rates. Neural Comput 9:1001-1013.

Jack JJB, Noble D, Tsien RW (1975) Electric current flow in excitable cells. Oxford: Clarendon.

Koch C (1999) Biophysics of computation: information processing in single neurons. Oxford: Oxford UP.

Koch C, Douglas R, Wehmeier U (1990) Visibility of synaptically induced conductance changes: theory and simulations of anatomically characterized cortical pyramidal cells. J Neurosci 10:1728-1744.

Koch C, Rapp M, Segev I (1996) A brief history of time (constants). Cereb Cortex 6:93-101.

Kuhn A, Rotter S, Aertsen A (2002) Subthreshold membrane potential fluctuations decrease with synaptic input rate in a simple model neuron with synaptic conductances. In: Proceedings of the 3rd Forum of European Neuroscience, 2002, 083.12.

Kuhn A, Aertsen A, Rotter S (2003) Higher-order statistics of input ensembles and the response of simple model neurons. Neural Comput 15:67-101.

London M, Meunier C, Segev I (1999) Signal transfer in passive dendrites with nonuniform membrane conductance. J Neurosci 19:8219-8233.

Matsumura M, Chen D, Sawaguchi T, Kubota K, Fetz EE (1996) Synaptic interactions between primate precentral cortex neurons revealed by spike-triggered averaging of intracellular membrane potentials in vivo. J Neurosci 16:7757-7767.

McCormick DA, Connors BW, Lighthall JW, Prince DA (1985) Comparative electrophysiology of pyramidal and sparsely spiny neurons of the neocortex. J Neurophysiol 54:782-806.

Monier C, Chavane F, Baudot P, Graham LJ, Frégnac Y (2003) Orientation and direction selectivity of synaptic inputs in visual cortical neurons: a diversity of combinations produces spike tuning. Neuron 37:663-680.

Moore CI, Nelson SB (1998) Spatio-temporal subthreshold receptive fields in the vibrissae representation of rat primary somatosensory cortex. J Neurophysiol 80:2882-2892.

Papoulis A (1991) Probability, random variables, and stochastic processes, Ed 3. Boston: McGraw-Hill.

Paré D, Shink E, Gaudreau H, Destexhe A, Lang E (1998) Impact of spontaneous synaptic activity on the resting properties of cat neocortical pyramidal neurons in vivo. J Neurophysiol 79:1450-1460.

Pei X, Volgushev M, Vidyasagar TR, Creutzfeld OD (1991) Whole cell recording and conductance measurements in cat visual cortex in vivo. NeuroReport 2:485-488.

Rapp M, Yarom Y, Segev I (1992) The impact of parallel fiber background activity on the cable properties of cerebellar Purkinje cells. Neural Comput 4:518-533.

Reyes A (2001) Influence of dendritic conductances on the input-output properties of neurons. Annu Rev Neurosci 24:653-675.

Rotter S, Diesmann M (1999) Exact digital simulation of time-invariant linear systems with applications to neuronal modeling. Biol Cybern 81:381-402. 
Rudolph M, Destexhe A (2003) A fast-conducting, stochastic integrative mode for neocortical neurons in vivo. J Neurosci 23:2466-2476.

Shadlen MN, Newsome WT (1994) Noise, neural codes and cortical organization. Curr Opin Neurobiol 4:569-579.

Shadlen MN, Newsome WT (1998) The variable discharge of cortical neurons: implications for connectivity, computation, and information coding. J Neurosci 18:3870-3896.

Softky WR, Koch C (1993) The highly irregular firing of cortical cells is inconsistent with temporal integration of random EPSPs. J Neurosci 13:334-350.

Tarczy-Hornoch K, Martin KAC, Jack JJB, Stratford KJ (1998) Synaptic interactions between smooth and spiny neurones in layer 4 of cat visual cortex in vitro. J Physiol (Lond) 508:351-363.
Tarczy-Hornoch K, Martin KAC, Stratford KJ, Jack JJB (1999) Intracortical excitation of spiny neurons in layer 4 of cat striate cortex in vitro. Cereb Cortex 9:833-843.

Tiesinga PHE, José JV, Sejnowski TJ (2000) Comparison of current-driven and conductance-driven neocortical model neurons with HodgkinHuxley voltage-gated channels. Physiol Rev 62:8413-8419.

Troyer TW, Miller KD (1997) Physiological gain leads to high ISI variability in a simple model of a cortical regular spiking cell. Neural Comput 9:971-983.

van Vreeswijk C, Sompolinsky H (1996) Chaos in neuronal networks with balanced excitatory and inhibitory activity. Science 274:1724-1726.

Volgushev M, Pernberg J, Eysel UT (2002) A novel mechanism of response selectivity of neurons in cat visual cortex. J Physiol (Lond) 540:307-320. 